Pacific Journal of Mathematics

DERIVATIONS AND COMMUTATIVITY OF RING

O. Chung, Jana Luth and Anthony N. Richoux 


\title{
DERIVATIONS AND COMMUTATIVITY OF RINGS
}

\author{
Lung O. Chung, Jiang Luh and Anthony N. Richoux
}

Let $R$ be a ring with center $C$ and Jacobson radical $J$. Let $\mathcal{I}$ be the additive group of all inner derivations of $R$ and $\mathscr{D}$ be an additive group of derivations of $R$ satisfying:

(i) For any $\partial \in \mathscr{D}$ and $\delta \in \mathscr{I},[\partial, \delta] \in \mathscr{D}$;

(ii) For any $x \in R, \partial x=0$ for all $\partial \in \mathscr{D}$ iff $x \in C$;

(iii) For any prime ideal $P$ in $R$ and any $x \in R, \partial x \in P$ for all $\partial \in \mathscr{D}$ iff $\delta x \in P$ for all $\delta \in \mathscr{J}$.

Suppose, for each $x \in R$ and $\partial \in \mathscr{D}$, there is a $p \in R$ which depends upon $x$ and $\partial$ such that $\partial x=(\partial x)^{2} p$. Then the nilpotent elements in $R$ are central and form an ideal $N$ in $R, R / N$ is a subdirect sum of division rings and commutative rings, and $R / J$ is a subdirect sum of division 'rings. Suppose further that, for each $x \in R$ and $\partial \in \mathscr{D}$, such a $p$ is a polynomial of $\partial x$ with integral coefficients. Then $R$ is necessarily commutative.

1. Introduction. A well known theorem of Wedderburn states that all finite division rings are fields. There are various generalizations of this theorem. Jacobson [4] proves that if, for each $x$ in a ring $R$, there is an integer $n(x)>1$ such that $x^{n(x)}=x$ then $R$ is commutative. Among others, Herstein [2] weakens Jacobson's condition by assuming $x^{n(x)}=x$ for only every commutator $x$ in $R$. In other directions of research, Putcha, Wilson and Yaqub [8] show that if, for each commutator $x$ in a ring $R$, there exists an integer $n(x)>1$ and a central element $z(x) \in R$ such that $x=x^{n(x)} z(x)$ then $R / J$ is a subdirect sum of division rings where $J$ denotes the Jacobson radical of $R$. Suppose further that such $n(x)=2$ for each commutator $x \in R$. Then $R$ is commutative. Recently, Ligh [6] proves that if, for each commutator $x$ in a ring $R$, there exists $p(x) \in R$ such that $x=x^{2} p(x)$ then $R / N$ is a subdirect sum of commutative rings and division rings where $N$ denotes the lower nil radical of $R$. In view of the fact that a commutator is simply in the image of an inner derivation, we introduce in this paper the notion of primary classes of derivations of a ring $R$. A primary class $\mathscr{D}$ of derivations of a ring $R$ is very much like the group $\mathscr{F}$ of all inner derivations of $R$ but it could be much smaller than $\mathscr{F}$.

In $\S 2$, we give some basic properties of primary classes of derivations.

In $\S 3$, we consider a ring $R$ having a primary class $\mathscr{D}$ of derivations and satisfying the following conditions: 
(A) For each $x \in R$ and $\partial \in \mathscr{D}$, there is a $p=p(x, \partial) \in R$ such that $\partial x=(\partial x)^{2} p$.

It is shown that the nilpotent elements in $R$ are central and hence form an ideal $N$ in $R$. Moreover, $R / N$ is a subdirect sum of division rings and commutative rings and $R / J$ is a subdirect sum of division rings. Thus Putcha, Wilson and Yaqub's and Ligh's results follow immediately.

In $\S 4$, we study a ring $R$ having a primary class $\mathscr{D}$ of derivations and satisfying the following condition:

(B) For each $x \in R$ and $\partial \in \mathscr{D}$, there is a polynomial $p(t)$ of $t$ with integral coefficients such that $\partial x=(\partial x)^{2} p(\partial x)$.

We prove that $R$ is necessarily commutative. This generalizes the celebrated result of Herstein cited in the first paragraph.

In $\S 5$, some remarks are given and open problems are proposed.

Throughout this paper, $R$ denotes an associative ring, $\mathscr{Z}$ the ring of integers, and $\mathscr{Q}$ the rational field. For $x, y \in R,[x, y]=$ $x y-y x$, and $\delta_{x}$ denotes the inner derivation: $r \rightarrow[x, r]$ for all $r \in R$. We recall that a mapping $\partial: R \rightarrow R$ is called a derivation of $R$ if $\partial(x y)=\partial x y+x \partial y$ and $\partial(x+y)=\partial x+\partial y$ for all $x, y \in R$.

2. Basic properties. Let $R$ be a ring, $C$ be its center and $\mathscr{J}$ be the additive group of all inner derivations of $R$. An additive group $\mathscr{D}$ of derivations of $R$ is said to be a primary class of derivations of $R$ if

(i) For any $\partial \in \mathscr{D}$ and $\delta \in \mathscr{F},[\partial, \delta] \in \mathscr{D}$;

(ii) For any $x \in R, \partial x=0$ for all $\partial \in \mathscr{D}$ if and only if $x \in C$;

(iii) For any prime ideal $P$ in $R$ and any $x \in R, \partial x \in P$ for all $\partial \in \mathscr{D}$ if and only if $\delta x \in P$ for all $\delta \in \mathscr{F}$.

It is clear that $\mathscr{I}$ itself is a primary class of derivations of $R$, (iii) $\Rightarrow$ (ii) for any prime ring $R$, and (i) is equivalent to that, for and $\partial \in \mathscr{D}$ and $x \in R, \delta_{\partial a} \in \mathscr{D}$.

It is also easy to see that $\partial x \in N$, the lower nil radical of $R$, for all $\partial \in \mathscr{D}$ if and only if $\delta x \in N$ for all $\delta \in \mathscr{F}$.

For convenience, we denote by $\mathscr{D}(R)$ the set of all elements $\partial x$ where $\partial \in \mathscr{D}$ and $x \in R$.

EXAMPLE 1. Let $F=G F\left(2^{n}\right)$ where $n \geqq 2$ and $w$ be a generator of the multiplicative group of $F$. Let $R$ be the ring of 2 by 2 matrices over $F$ and $\mathscr{D}$ be the set of inner derivations $\delta_{x}$ where $x$ is of the form $\alpha\left(\begin{array}{ll}0 & 1 \\ w & 0\end{array}\right)+\beta\left(\begin{array}{ll}0 & 1 \\ w^{2} & 0\end{array}\right), \alpha, \beta \in F$. It is easy to see that $\left[\partial, \partial^{\prime}\right]=0$ for all $\partial, \partial^{\prime} \in \mathscr{D}$ and $\mathscr{D}$ forms a primary class of deriva- 
tions of $R$ which is properly contained in $\mathscr{F}$, the group of all inner derivations of $R$.

EXAMPLE 2. Let $F$ be a field and $R=F[x, y]$ be the free algebra generated by $x$ and $y$ over $F$. Let $\mathscr{D}$ be the Lie ideal of $\mathscr{I}$ generated by $\delta_{x}$ and $\delta_{y}$. Then $\mathscr{D}$ is a primary class of derivations of $R . \mathscr{D} \neq \mathscr{I}$ since $\delta_{x^{2}}, \delta_{x y}, \delta_{y^{2}}, \delta_{x^{3}}, \cdots$, are not in $\mathscr{D}$.

The authors, however, do not know whether a primary class of derivations of a ring $R$ is necessarily contained in the group of all inner derivations of $R$.

Proposition 2.1. Let $R$ be a ring and $R^{*}$ be a prime ring which is a homomorphic image of $R$ under a homomorphism $\phi$. Suppose $\mathscr{D}$ is a primary class of derivations of $R$. For each $\partial \in \mathscr{D}$, define $\partial^{*}: R^{*} \rightarrow R^{*}$ by $\partial^{*}(\phi(x))=\phi(\partial x)$ for all $x \in R$. Then the set $\mathscr{D}^{*}$ of all $\partial^{*}$, where $\partial \in \mathscr{D}$, forms a primary class of derivations of $R^{*}$.

Proof. For $\partial \in \mathscr{D}, \partial^{*}$ is well defined since $\phi(x)=\phi(y) \Rightarrow x-y \epsilon$ $\operatorname{Ker} \phi \Rightarrow \delta(x-y) \in \operatorname{Ker} \phi$ for all $\delta \in \mathscr{J} \Rightarrow \partial(x-y) \in \operatorname{Ker} \phi$ for all $\partial \epsilon$ $\mathscr{D} \Rightarrow \phi(\partial x)=\phi(\partial y)$. Here we use the fact that $\operatorname{Ker} \phi$ is a prime ideal in $R$. It is readily to verify that $\mathscr{D}^{*}$ forms an additive group of derivations of $R^{*}$ and that $\mathscr{D}^{*}$ satisfies the condition (i).

We observe that for $\phi(c) \in R^{*}, \phi(c)$ is central in $R^{*} \Leftrightarrow \phi(c) \phi(x)=$ $\phi(x) \phi(c)$ for all $x \in R \Leftrightarrow[c, x] \in \operatorname{Ker} \phi$ for all $x \in R \Leftrightarrow \partial c \in \operatorname{Ker} \phi$ for all $\partial \in \mathscr{D} \Leftrightarrow \partial^{*}(\phi(c))=0$ for all $\partial^{*} \in \mathscr{D}^{*}$. Thus the condition (ii) holds for $\mathscr{D}^{*}$.

To see (iii), let $P^{*}$ be a prime ideal in $R^{*}$. Set $P=$ $\left\{x \in R \mid \phi(x) \in P^{*}\right\} . \quad P$ is then a prime ideal in $R$. For $x \in R$, $\partial^{*}(\phi(x)) \in P^{*}$ for all $\partial^{*} \in \mathscr{D}^{*} \Leftrightarrow \phi(\partial x) \in P^{*}$ for all $\partial \in \mathscr{D} \Leftrightarrow \partial x \in P$ for all $\partial \in \mathscr{D} \Leftrightarrow \delta x \in P$ for all $\delta \in I \Leftrightarrow \phi(\delta x) \in P^{*}$ for all $\delta \in \mathscr{I} \Leftrightarrow \delta^{*}(\phi(x)) \in P^{*}$ for all inner derivations $\delta^{*}$ of $R^{*}$. Thus (iii) holds for $\mathscr{D}^{*}$.

CoRollaRy 2.2. Let $R$ be a ring and $P$ be a prime ideal in $R$. Suppose $\mathscr{D}$ is a primary class of derivations of $R$. For each $\partial \in \mathscr{D}$, define $\partial^{*}: R / P \rightarrow R / P$ by $\partial^{*}(x+P)=\partial x+P$ for all $x \in R$. Then the set $\mathscr{D}^{*}$ of all $\partial^{*}$, where $\partial \in \mathscr{D}$, forms a primary class of derivations of $R / P$.

Corollary 2.3. In Corollary 2.2, if $P$ is replaced by the lower nil radical $N$ of $R$, the statement remains true. 
Proof. Use the fact that $N$ is the intersection of all prime ideals in $R$.

COROLlary 2.4. In Corollary 2.2, if $P$ is replaced by the Jacobson radical $J$ of $R$ the statement remains true.

Proof. Using the fact that $J$ is the intersection of all prime ideals in $R$ containing $J$, one can show that for each $\partial \in \mathscr{D}, \partial^{*}$ is well defined and that the conditions (i)-(iii) follow immediately for $\mathscr{D}^{*}$.

3. Primary class satisfying (A). Throughout this sections, we assume that $R$ is a ring having a primary class $\mathscr{D}$ of derivations of $R$. Furthermore, we assume that $\mathscr{D}$ satisfies the condition (A) stated in the introduction.

Under condition (A), it is clear that zero is the only nilpotent element in $\mathscr{D}(R)$. We shall show first that all nilpotent elements in $R$ are central. We begin with

Lemma 3.1. Let $x \in R$ and $\partial \in \mathscr{D}$. If $x^{2}=0$, then $x \partial x+\partial x x=0$ and $[\partial x, x]=0$.

Proof. $\partial x^{2}=0$ implies $x \partial x+\partial x x=0$, consequently, $[\partial x, x]^{2}=$ $(\partial x x-x \partial x)^{2}=\partial x x \partial x x-x(\partial x)^{2} x+x \partial x x \partial x=0$. Since $[\partial x, x] \in \mathscr{D}(R)$ by condition (i), in the definition of a primary class of derivations, $[\partial x, x]=0$.

Lemma 3.2. Let $x, z \in R$ and $\partial \in \mathscr{D}$. If $x^{2}=0$ and $[\partial z, x]=0$, then $[\partial z, x y]=0$ for all $y \in R$.

Proof. Since $[\partial z, x y x]=\partial z x y x-x y x \partial z=x[\partial z, y] x \in \mathscr{D}(R)$ and its square is zero, we have $x[\partial z, y] x=0$. Thus, $[\partial z, x y]=$ $\partial z x y-x y \partial z=x[\partial z, y]$ is a nilpotent element in $\mathscr{D}(R)$ and hence $[\partial z, x y]=0$.

Let us recall that the characteristic of an element $x \in R$, char $x$, is the order of $x$ in the additive group of $R$.

LEMma 3.3. Let $x \in R$ and $\partial \in \mathscr{D}$. If $x^{2}=0$ and if $\operatorname{char}(x \partial x) \neq 2$, then $\partial x=0$.

Proof. By Lemma 3.1, $2 x \partial x=0$ and hence $x \partial x=\partial x x=0$. By expanding $\partial(x \partial x)=0$, we obtain $(\partial x)^{2}+x \partial^{2} x=0$. Pre-multiplying by $\partial x$ yields $(\partial x)^{3}=0$. Thus $\partial x=0$. 
Lemma 3.4. Let $x \in R$ and $\partial \in \mathscr{D}$. If $x^{2}=0$ and $\operatorname{char}(x \partial x)=2$, then $2 \partial x=0$ and $\partial x \in C$.

Proof. Similar to the proof of Lemma 3.3, we obtain $2 \partial x=0$. Since $[\partial x, x]=0$, by Lemma $3.2, x[\partial x, y]=[\partial x, x y]=0$ for all $y \in R$. It follows that

$$
0=\partial(x[\partial x, y])=\partial x[\partial x, y]+x\left[\partial^{2} x, y\right] .
$$

But $0=\partial([\partial x, x])=(\partial x)^{2}+x \partial^{2} x+\partial^{2} x x+(\partial x)^{2}=x \partial^{2} x+\partial^{2} x x=\left[\partial^{2} x, x\right]$ and hence by Lemma 3.2, $x\left[\partial^{2} x, y\right]=\left[\partial^{2} x, x y\right]=0$ for all $y \in R$. Therefore, $\partial x[\partial x, y]=0$ and it follows that $[\partial x, y]^{2}=\partial x y[\partial x, y]=$ $\partial x y(\partial x y+y \partial x)=(\partial x)^{2} y^{2}+(\partial x)^{2} y^{2}=0$. Thus, $[\partial x, y]=0$ for all $y \in R$ and $\partial x \in C$.

Lemma 3.5. Let $x, y \in R$ and $\partial \in \mathscr{D}$. If $x^{2}=0$ and $[\partial y, x]=0$, then $\partial_{1} x \partial y \in C$ for all $\partial_{1} \in \mathscr{D}$.

Proof. By Lemma 3.2, $x[\partial y, z]=[\partial y, x z]=0$ for all $z \in R$ and hence $0=\partial_{1}(x[\partial y, z])=\partial_{1} x[\partial y, z]+x\left[\partial_{1} \partial y, z\right]$. Since $\partial_{1}[\partial y, x]=0$, $\left[\partial_{1} \partial y, x\right]=0$ by Lemmas 3.3 and 3.4 and $\left[\partial_{1} \partial y, x z\right]=x\left[\partial_{1} \partial y, z\right]=0$ for all $z \in R$ by Lemma 3.2. Thus, $0=\partial_{1} x[\partial y, z]=\left[\partial_{1} x \partial y, z\right]$ for all $z \in R$ and $\partial_{1} x \partial y \in C$.

LEMma 3.6. Let $x \in R$. If $x^{2}=0$ then $\partial_{2} x \partial_{1} \partial y \in C$ for all $\partial, \partial_{1}, \partial_{2} \in \mathscr{D}$ and $y \in R$.

Proof. Noting that $\partial x \in C$ for all $\partial \in \mathscr{D}$, we have $[\partial y, x]=$ $\delta_{\partial y}(x) \in C$ which implies $\left[\partial_{1} \partial y, x\right]=\partial_{1}[\partial y, x]=0$. Thus, by Lemma $3.5, \partial_{2} x \partial_{1} \partial y \in C$.

Lemma 3.7. Let $x \in R$. If $x^{2}=0$ then $\partial_{1} x \partial^{2} y=0$ for all $\partial, \partial_{1} \in \mathscr{D}$ and $y \in R$.

Proof. $\partial_{1} x x \partial^{2} y=\partial_{1} x \partial^{2}(x y) \in C$ by Lemmas 3.3, 3.4, and 3.6. Hence $0=\partial_{1}\left(\partial_{1} x x \partial^{2} y\right)=\left(\partial_{1} x\right)^{2} \partial^{2} y$. By the condition (A), $\partial_{1} x \partial^{2} y=0$.

Lemma 3.8. Let $x, y \in R$ and $\partial \in \mathscr{D}$. If $\partial x \in C$ and $(\partial x)^{2} y \in C$ then $\partial x y \in C$.

Proof. Suppose $\partial x=(\partial x)^{2} p$ where $p \in R$. Then, for any $z \in R$, $0=p\left[(\partial x)^{2} y, z\right]=p(\partial x)^{2}[y, z]=(\partial x)[y, z]=[\partial x y, z]$.

LemmA 3.9. Let $x \in R$. If $x^{2}=0$ then $\partial_{2} x\left(\partial_{1} x \partial y+\partial x \partial_{1} y\right) \in C$ for all $\partial, \partial_{1}, \partial_{2} \in \mathscr{D}$ and $y \in R$. 
Proof. Let $u=\partial_{2} x\left(\partial_{1} x \partial y+\partial x \partial_{1} y\right)$. By Lemma 3.6, $\partial_{2} x \partial_{1} \partial(x y) \in C$, i.e., $u+\partial_{2} x x \partial_{1} y \in C$. Since $\partial_{2} x, \partial_{2} x \partial_{1} \partial y \in C$, we have $\left[\partial_{2} x x \partial_{1} \partial y, x\right]=0$ and hence $[u, x]=0$. For any $z \in R,[u, z]=\left(\delta_{\partial\left(\partial_{2} x \partial_{1} x y\right)}+\delta_{\partial_{1}(\partial x \partial x y)}\right)(z) \in$ $\mathscr{D}(R)$ and hence $[u, x z x]=x[u, z] x \in \mathscr{D}(R)$. Since $x[u, z] x$ is nilpotent, $x[u, z] x=0$ and $x[u, z]$ is nilpotent. But $x[u, z]=[u, x z] \in \mathscr{D}(R)$. So $x[u, z]=0$. Noting that $\partial_{2} u=\partial_{2} x\left(\partial_{1} x \partial_{2} \partial y+\partial x \partial_{2} \partial_{1} y\right) \in C$ by Lemma 3.6, we obtain $0=\partial_{2}(x[u, z])=\partial_{2} x[u, z]=\left[\partial_{2} x u, z\right]$. So $\partial_{2} x u \in C$. By Lemma 3.8, $u \in C$.

LEMMA 3.10. Let $x \in R$. If $x^{2}=0$ then $\partial_{1} x \partial_{1} \partial y=0$ for all $\partial, \partial_{1} \in \mathscr{D}$ and $y \in R$.

Proof. Let $\partial \in \mathscr{D}$. Suppose $\partial x=0$. Then according to Lemma $3.9, \partial_{2} x \partial_{1} x \partial y \in C$ for all $\partial_{1}, \partial_{2} \in \mathscr{D}$ and $y \in R$. Particularly, $\left(\partial_{1} x\right)^{2} \partial y \in C$ and by Lemma 3.8, $\partial_{2} x \partial y \in C$. Thus, $0=\partial_{1}\left(\partial_{1} x \partial y\right)=\partial_{1} x \partial_{1} \partial y$.

Now, suppose $\partial x \neq 0$. Then by Lemma 3.9, $\partial_{1}\left(\partial_{2} x \partial_{1} x y+\partial_{2} x \partial y \partial_{1} y\right)=0$ or $\partial_{2} x \partial_{1} x \partial_{1} y=0$ for all $\partial_{1}, \partial_{2} \in \mathscr{D}$ and $y \in R$. Particularly, $\left(\partial_{1} x\right)^{2} \partial_{1} \partial y=0$. Using the condition (A) again $\partial_{1} x \partial_{1} \partial y=0$.

Lemma 3.11. Let $x \in R$. If $x^{2}=0$ then $\partial x \partial y \in C$ for all $\partial \in \mathscr{D}$ and $y \in R$.

Proof. By Lemma 3.10, $\partial x \partial \partial_{1}(y x)=0$ or $0=\partial x\left(\partial \partial_{1} y x+\partial_{1} y \partial x+\right.$ $\left.\partial y \partial_{1} x\right)=\partial x\left(\partial_{1} y \partial x+\partial y \partial_{1} x\right)$. Consequently, $\partial_{1}(\partial x \partial(y x))=\partial x\left(\partial_{1} \partial y x+\partial_{1} y \partial x+\right.$ $\left.\partial y \partial_{1} x\right)=\partial x \partial_{1} \partial y x \in \mathscr{D}(R)$ while its square is zero by Lemma 3.6. Since zero is the only nilpotent element in $\mathscr{D}(R) \partial x \partial_{1} \partial y x=0$, and since $\partial x \partial_{1} \partial y \in C$ and $\partial x \in C, \quad 0=\partial\left(\partial x \partial_{1} \partial y x\right)=\left(\partial x \partial_{1} \partial y\right) \partial x=(\partial x)^{2} \partial_{1} \partial y$. Applying the condition (A), we get $\partial x \partial_{1} \partial y=0$, i.e., $\partial_{1}(\partial x \partial y)=0$. Since $\partial_{1}$ is arbitrary in $\mathscr{D}, \partial x \partial y \in C$ as we desired.

Now we are in the position to prove the following

Proposition 3.12. All nilpotent elements in $R$ are central and hence form an ideal in $R$.

Proof. Suppose $x \in R$ and $x^{n}=0$. We proceed by induction on n. Suppose $n=2$. By Lemma 3.11, $\partial x \partial(x y) \in C$ for all $\partial \in \mathscr{D}$ and $y \in R$. From $[\partial x \partial(x y), x]=0$, we arrive $\left[(\partial x)^{2} y+\partial x x \partial y, x\right]=0$. This implies that $\left[(\partial x)^{2} y, x\right]=0$ by Lemma 3.11, i.e., $\left[y,(\partial x)^{2} x\right]=0$ and $(\partial x)^{2} x \in C$. Thus, $0=\partial\left((\partial x)^{2} x\right)=(\partial x)^{3}$ and consequently, $\partial x=0$. This is true for all $\partial \in \mathscr{D}$, so $x \in C$.

Now we assume $x^{n+1}=0$, where $n>1$. Since $\left(x^{2}\right)^{n}=\left(x^{3}\right)^{n}=0$, $x^{2}$ and $x^{3}$ are central by the induction hypothesis. Let $\partial \in \mathscr{D}$. 
$0=\partial x^{2}=x \partial x+\partial x x$ and $0=\partial x^{3}=x^{2} \partial x$ yield $[\partial x, x]^{2}=0$. Since $[\partial x, x] \in \mathscr{D}(R),[\partial x, x]=0$. Noting that $0=x\left(\partial x^{2}\right)=x \partial x x$ we obtain $(x \partial x)^{2}=x[\partial x, x] \partial x=0$. Thus, by the induction hypothesis, $x \partial x \in C$ and, consequently, $0=\partial(x \partial x)=(\partial x)^{2}+x \partial^{2} x$. Pre-multiplaying by $x$ and $(\partial x)^{2}$ respectively and by noting that $0=\partial\left(x^{2} \partial x\right)=\partial x^{2} \partial x+x^{2} \partial^{2} x=$ $x^{2} \partial^{2} x$, we obtain $x(\partial x)^{2}=0$ and hence $(\partial x)^{4}=0$. Thus $\partial x=0$ for all $\partial \in \mathscr{D}$ and $x \in C$. This completes the proof.

Corollary 3.13. Let $A$ be a ring. Suppose for each commutator $x \in A$, there exists a $p(x) \in A$ such that $x=x^{2} p(x)$. Then all nilpotent elements in $A$ are central and form an ideal in $A$.

REMARK. The proof of Proposition 3.12 can be simplified slightly by transfinite method. Let $\phi_{i}$ be epimorphisms of $R$ onto prime rings $R_{i}$ with $\bigcap_{i} \operatorname{Ker} \phi_{i}=N$. First show that each $R_{i}$ has no nonzero nilpotent elements. Then for each nilpotent element $x \in R$, $\phi_{i}(x)=0$ and consequently $\phi_{i}(\partial x)=0$ for all $i$. This means that $\partial x \in N$ and so $\partial x=0$ for all $\partial \in \mathscr{D}$.

Proposition 3.14. Suppose $R$ is a prime ring. Then $R$ is either a commutative ring or a division ring.

Proof. We should note that $R$ has no zero divisors. For $x y=0$ implies $(y R x)^{2}=0$. Since $R$ has nonzero nilpotent elements according to Proposition 3.12, $y R x=0$ and whence $x=0$ or $y=0$.

Suppose now to the contrary that $R$ is neither a commutative ring nor a division ring. Then $\mathscr{D}(R) \neq(0)$. Let $0 \neq \partial x \in \mathscr{D}(R)$ and $\partial x=(\partial x)^{2} p$, where $p \in R$. Then, for any $y \in R, \partial x(y-\partial x p y)=0$ yields $y=\partial x p y$, so $\partial x p$ is a left identity in $R$. By Proposition 3.12, all nilpotent elements in $R$ are central so all idempotent elements in $R$ are also central and, particularly, $\partial x p \in C$. Therefore, $\partial x p$ is a two-sided identity element in $R$ which will be denoted by 1 . This shows that, for $\partial x \in \mathscr{D}(R)$, either $\partial x=0$ or $\partial x$ is invertible.

Since we also assume that $R$ is not a division ring, there exists $0 \neq a \in R$ which is not invertible. Suppose $a \in C$. Then, for any $\partial \in \mathscr{D}, \partial(a x)=\partial a x+a \partial \partial x=a \partial x \neq 0$ for some $x \in R$. Thus $\partial(a x)$ is invertible so is $a$, a contradiction. Therefore $a \notin C$. Let $0 \neq \partial \in \mathscr{D}$ and let $b \in R, \partial b \neq 0$. Observe $\partial\left(a^{2} b\right)=\partial a^{2} b+a^{2} \partial b$. Suppose $\partial a^{2}$ is not invertible. Then $\partial a^{2}=0$ and $\partial\left(a^{2} b\right)=a^{2} \partial b \neq 0$. Hence $\partial\left(a^{2} b\right)$ is invertible, so is $a$, again contradicting the choice of $a$. Thus, $\partial a^{2}$ must be invertible for each nonzero $\partial \in \mathscr{D}$. Likewise $\partial a^{3}$ is invertible for each nonzero $\partial \in \mathscr{D}$. Let $u$ and $v$ be respectively the inverses of $\partial a^{2}$ and $\partial a^{3}$. Then $\partial\left(a^{2} u-a^{3} v\right)=\partial a^{2} u+a^{2} \partial u-\partial a^{3} v-a^{3} \partial v=$ $a^{2}(\partial u-a \partial v)$ which is not invertible since $a$ is not. Hence 
$\partial\left(a^{2} u-a^{3} v\right)=0$. However, $a^{2} u-a^{3} v=a^{2}(u-a v) \neq 0$ since $a \neq 0$ and $a$ is not a unit. It follows that $\partial\left(\left(a^{2} u-a^{3} v\right) b\right)=\partial\left(a^{2} u-a^{3} v\right) b+$ $\left(a^{2} u-a^{3} v\right) \partial b=\left(a^{2} u-a^{3} v\right) \partial b \neq 0$. Consequently, $\partial\left(\left(a^{2} u-a^{3} v\right) b\right)$ and hence $a^{2} u-a^{3} v$ are invertible. This is clearly impossible since $a$ is not invertible. This completes the proof.

THEOREM 3.15. Let $R$ be a ring with lower nil radical $N$. Then $R / N$ is a subdirect sum of division rings and commutative rings.

Proof. From Corollary 2.3, $R / N$ is a ring having a primary class of derivations $\mathscr{D}^{*}$ satisfying the condition (A). It is known by Proposition 3.12 that $N$ is the set of all nilpotent elements in $R, R / N$ has no nilpotent elements $\neq 0$, and $R / N$ is a subdirect sum of prime rings $R_{i}$. By Proposition 2.1, each $R_{i}$ possesses a primary class of derivations which satisfies the condition (A). Each $R_{i}$ is either a division ring or a commutative ring by Proposition 3.14.

CoRollary 3.16 (Ligh). Suppose, for each commutator $x \in A$, there exists $p(x) \in A$ such that $x=x^{2} p$. Then $A / N$ is a subdirect sum of division rings and commutative rings, where $N$ is the lower nil radical of $A$.

THEOREM 3.17. Let $R$ be a ring with Jacobson radical J. Then $R / J$ is a subdirect sum of division rings.

Proof. $R / J$ is a subdirect sum of primitive rings. Each subdirect summand is a division ring by Proposition 3.14 and the fact that commutative primitive rings are fields.

Corollary 3.18 (Putcha, Wilson, Yaqub). Let $A$ be a ring. Suppose, for each commutator $x \in A$, there are a central element $z \in A$ and an integer $n>1$ such that $x=x^{n} z$. Then $A / J$ is a subdirect sum of division rings, where $J$ denotes the Jacobson radical of $A$.

In the balance of this section, we shall consider those rings $R$ having a primary class $\mathscr{D}$ of derivations and satisfying a rather stronger condition than the condition (A), namely,

(C) For each $x \in R$ and $\partial \in \mathscr{D}$, there exists an element $z \in C$ such that $\partial x=(\partial x)^{2} z$.

THEOREM 3.19. Let $R$ be a ring having a primary class $\mathscr{D}$ of 
derivations and satisfying the condition (C). Then $R$ is commutative.

Proof. By Theorem 3.15, $R / N$ is a subdirect sum of rings $R_{i}$, where each $R_{i}$ is either a commutative ring or a division ring. Suppose $R_{i}$ is a division ring. Let $\mathscr{D}_{i}^{*}$ be the primary class of derivations of $R_{i}$ induced from $\mathscr{D}$. For $x \in R_{i}$ and $\partial^{*} \in \mathscr{D}_{i}, \partial^{*} x=$ $\left(\partial^{*} x\right)^{2} z$ where $z$ lies in the center of $R_{i}$. If $\partial^{*} x \neq 0$, then $\partial^{*} x$ has inverse $z$ which is central. Thus $\partial^{*} x$ is central for all $\partial^{*} \in \mathscr{D}_{i}^{*}$ and $x \in R_{i}$. If the characteristic of $R_{i}$ is 2 then, for $\partial^{*} \in \mathscr{D}_{i}^{*}, \partial^{*}\left(x^{2}\right)=$ $2 x \partial^{*} x=0$. Thus $x^{2}$ is central for all $x \in R_{i}$. By a well known result of Kaplansky (see [4], p. 219), $R_{i}$ is commutative. Now suppose the characteristic of $R_{i}$ is not 2. Then, for $0 \neq x \in R_{i}$, let $y$ be the inverse of $x$. We have, for $\partial^{*} \in \mathscr{D}_{i}^{*}, 0=\partial^{*}(x y)=\partial^{*} x y+$ $x \partial^{*} y$ and $0=\partial^{* 2}(x y)=2 \partial^{*} x \partial^{*} y$ which yield that $0=\partial^{*} x\left(\partial^{*} x y+x \partial^{*} y\right)=$ $\left(\partial^{*} x\right)^{2} y$. Thus, $\partial^{*} x=0$ for all $\partial^{*} \in \mathscr{D}_{i}^{*}$ and $x \in R_{i}$. $R_{i}$ is therefore commutative.

$R / N$ being a subdirect sum of commutative rings is commutative. To see that $R$ is commutative, let $x, y \in R$. Then $x y-y x \in N$ yields $\partial x \in N$ and hence $\partial x=0$ for all $\partial \in \mathscr{D}$ and $x \in R$. Thus $R$ is commutative.

CoRollary 3.20 (Putcha, Wilson, Yaqub). Let $R$ be a ring. Suppose, for each commutator $x \in R$, there exists a central element $z$ which depends upon $x$, such that $x=x^{2} z$. Then $R$ is commutative.

4. Primary class satisfying (B). Let us first list a useful result which will be used in the sequel of this section.

LEMMA 4.1. Let $F$ be a division ring of characteristic $p \neq 0$ and $C$ be the center of $F$. Suppose $a \in F \backslash C$ and $a^{p^{n}}=a$ for some integer $n \geqq 1$. Then there exists an $x \in F$ such that $x a x^{-1}=a^{i} \neq a$ for some integer $i$.

Proof. (See [3], p. 70.)

LEMmA 4.2. Let $R$ be a division ring and $\mathscr{D}$ be a primary class of derivations of $R$. Suppose, for each $a \in R$ and $\partial \in \mathscr{D}$, there exists an integer $n>1$ which depends upon $a$ and $\partial$ such that $\partial a=(\partial a)^{n}$. Then $R$ is a field.

Proof. Suppose to the contrary that $R$ is not a field. Let $a \in R \backslash C$, where $C$ denotes the center of $R$. Then there exists $\partial \in \mathscr{D}$ 
such that $\partial a \neq 0$, and $(\partial a)^{m}=\partial a$. Let $P$ be the prime field of $R$. Since $2 \partial \in \mathscr{D}, 2 \partial a=(2 \partial a)^{n}=2^{n}(\partial a)^{n}$ for some integer $n>1$. Let $q=(m-1)(n-1)+1$. One can see easily that $(2 \partial a)^{q}=2 \partial a$ and $(\partial a)^{q}=\partial a$. Hence $2^{q}=2$ and $R$ is of nonzero characteristic $p$ say.

We shall show now that, for each $0 \neq \partial \in \mathscr{D}$, there is an $a \in R$ such that $\partial a \notin C$. suppose not. Then $[\partial x, y]=0$ for all $x, y \in R$ and $\partial_{1} \partial(x)=0$ for all $x \in R, \partial_{1} \in \mathscr{D}$. If $p \neq 2$, we have $0=\partial\left(\partial x^{2}\right)=$ $\partial(2 x \partial x)=2(\partial x)^{2}$ and consequently $\partial x=0$ for all $x \in R$, a contradiction. Thus we may assume now $p=2$. Since, for each $x \in R$, $\partial\left(x^{2}\right)=2 x \partial x=0$, we have, for all $x, y \in R, 0=\left[\partial\left(x x^{2}\right), y\right]=\partial x\left[x^{2}, y\right]$. If $\partial x \neq 0$ then $\left[x^{2}, y\right]=0$ for all $y \in R$ and $x^{2} \in C$. If $\partial x=0$, then since, for all $y, z \in R, 0=[\partial(x z), y]=\partial z[x, y],[x, y]=0$ for all $y \in R$ and hence $x \in C$. Thus in either case, $x^{2} \in C$. Using again the result of Kaplansky cited in the proof of Theorem 3.19, we obtain the commutativity of $R$, a contradiction. This proves the assertion that, for each $0 \neq \partial \in \mathscr{D}$, an element $a \in R$ exists with $\partial a \notin C$. For this $\partial a,(\partial a)^{m}=\partial a$ for some integer $m>1$ and hence $\partial a$ is algebraic over $P$ and hence $(\partial \alpha)^{p^{k}}=\partial \alpha$ for some integer $k>0$. By Lemma 4.1, there is an $x \in R$ such that $x \partial a x^{-1}=(\partial a)^{i} \neq \partial a$, i.e., $x \partial a=$ $(\partial a)^{i} x \neq \partial a x$. Thus, $[\partial a, x] \neq 0$ and $[\partial a, x] \partial \alpha=\partial a x \partial a-x(\partial a)^{2}=$ $(\partial a)^{i}(\partial a x-x \partial a)=(\partial a)^{i}[\partial a, x]$. However, by the hypothesis, $[\partial a, x]^{h}=$ $[\partial a, x]$ for some integer $h>1$. Thus the ring $S=\left\{\sum_{\alpha_{i j}}(\partial a)^{i}[\partial a, x]^{j} \mid\right.$ $\left.\alpha_{i j} \in P\right\}$ is a finite division ring. By the Wedderburn theorem for finite division rings, $S$ is a field. Consequently $\partial \alpha[\partial \alpha, x]=[\partial \alpha, x] \partial \alpha$, a contradiction.

In the balance of this section, we assume $R$ is a ring having a primary class $\mathscr{D}$ of derivations. Also we assume $\mathscr{D}$ satisfies the condition (B) stated in the introduction.

Perhaps we should note that the condition (B) implies the condition (A) and hence we can use the results in $\S 3$ freely in the sequel of this section.

\section{LemMa 4.3. If $R$ is a division ring then $R$ is a field.}

Proof. Suppose the characteristic of $R$ is not zero, and $P$ is the (finite) prime field of $R$. Since each $\partial a \in \mathscr{D}(R)$ is algebraic over $P$, there exists an integer $m>1$ such that $(\partial a)^{m}=\partial a$. By Lemma $4.2, R$ is commutative. Suppose the characteristic of $R$ is zero. Suppose to the contrary that $R$ is not commutative and $a \in R$ is not central. Then there is $\partial \in \mathscr{D}$ with $\partial a \neq 0$. For each prime number $p \in \mathscr{Z}$, there exists $f_{p}(t) \in \mathscr{Z}[t]$ such that $(p \partial \alpha)^{2} f_{p}(p \partial \alpha)=p \partial \alpha$. Thus $p \partial a f_{p}(p \partial a)-1=0$. Let $g_{p}(t) \in \mathscr{Y}[t]$ be a polynomial with least degree such that $p \partial a g_{p}(\partial a)-1=0$. We claim that $p t g_{p}(t)-1$ is 
irreducible over $\mathscr{F}$ and hence it is also irreducible over $\mathscr{Q}$ by the Gauss lemma. Suppose $p t g_{p}(t)-1=h(t) k(t)$, where $h(t), k(t) \in \mathscr{Z}[t]$, $h(\partial a)=0$ and $k(t) \neq \pm 1$. By comparing the constant terms of $p t g_{p}(t)-1$ and $h(t) k(t)$, one can see that $k(t)$ is not a constant and the degree of $k(t)$ is $n$ say. Let $\alpha, \beta, \gamma$ be respectively the leading coefficients of $k(t), h(t)$, and $g_{p}(t)$. Then $\alpha \beta=p \gamma$. Since $h(t) k(t) \equiv$ $-1(\bmod p), k(t)$ is a constant polynomial modulo $p$ and $p \mid \alpha$. Thus $\operatorname{deg}\left(g_{p}(t)-\alpha p^{-1} t^{n-1} h(t)\right)<\operatorname{deg} g_{p}(t)$ and since $p \partial \alpha g_{p}(\partial a)=1$ and $h(\partial a)=0$, $p \partial a\left(g_{p}(\partial \alpha)-\alpha p^{-1}(\partial \alpha)^{n-1} h(\partial a)\right)-1=0$. This contradicts the choice of $g_{p}(t)$. Therefore, for each prime number $p, p t g_{p}(t)-1$ is irreducible over $\mathscr{Q}$ and has $\partial a$ as a root. All these polynomials therefore must be divisible by each other over $\mathbb{Q}$. This clearly is impossible, and the lemma is proved.

From Proposition 3.14 and Lemma 4.3, we immediately have the following

LEMMA 4.4. If $R$ is a prime ring then $R$ is commutative.

Now we are in the position to prove our main result of this section.

THEOREM 4.5. Let $R$ be a ring having a primary class $\mathscr{D}$ of derivations which satisfies the condition (B). Then $R$ is commutative.

Proof. By Corollary 2.4, $\mathscr{D}$ induces a primary class $\mathscr{D}^{*}$ of derivations of $R / J$, where $J$ denotes the Jacobson radical of $R$. Being semisimple, $R / J$ is a subdirect sum of primitive rings. According to Lemma 4.4, each subdirect summand is commutative. Hence $R / J$ is commutative. Note that $J$ is the intersection of all prime ideals of $R$ which contain $J$. Since for all $x \in R$ and $\delta \in \mathscr{F}$, the group of all inner derivations of $R, \delta x \in J$, we have $\partial x \in J$ for all $\partial \in \mathscr{D}$. From the condition $(\mathrm{B}), \partial x=(\partial x)^{2} p(\partial x)$ for some $p(t) \in \mathscr{z}[t]$. Let $e=\partial x p(\partial x)$. Clearly, $e \in J$ and $\partial x=\partial x e$. Let $f$ be the quasi-invere of $e$. Then $0=\partial x e(e+f-e f)=\partial x e=\partial x$. Thus, $\partial x=0$ for all $x \in R$ and $\partial \in \mathscr{D}$ and $R$ is commutative.

The following corollary which generalizes the celebrated result of Herstein cited in the introduction is an immediate consequence of Theorem 4.5.

COROLlary 4.6. Let $A$ be a ring. If, for each commutator $x \in A$, there exists a polynomial $p(t) \in \mathscr{F}[t]$ such that $x=x^{2} p(x)$, then 
$A$ is commutative.

5. Some remarks and open problems. We have seen that, in many imposed conditions which imply the commutativity or "near" commutativity of rings $R$, general elements in $R$ may often be replaced by the elements in $\mathscr{D}(R)$, where $\mathscr{D}$ is a primary class of derivations of $R$. In view of this, we would raise the question as to what extent can we replace elements of $R$ or commutators by elements of $\mathscr{D}(R)$ and still yield the same or similar results? Thus a wide field of questions on commutativity is open. For example, one problem immediately comes to our minds: By the results given by Herstein, Martindale, Utumi and many others ([1], [3], [7], [9], etc.), we would like to propose the following open problems:

Let $\mathscr{D}$ be a primary class of derivations of a ring $R$ and $C$ be the center of $R$.

(1) Suppose, for each $\partial x \in \mathscr{D}(R)$, there exists a $p \in R$ which depends upon $\partial x$, such that $\partial x-(\partial x)^{2} p \in C$. Do the nilpotent elements in $R$ form an ideal $N$ ? If so, is $R / N$ a subdirect sum of commutative rings and division rings?

(2) Suppose, for each $\partial x \in \mathscr{D}(R)$, there exists a polynomial $p(t) \in \mathscr{L}[t]$ which depends upon $\partial x$, such that $\partial x-(\partial x)^{2} p(\partial x) \in C$. Does $\mathscr{D}(R) \subseteq C$ ?

Ligh has recently extended many commutativity theorems for rings including the one by Putcha, Wilson and Yaqub to near rings or d.g. near rings. For this reason we would like to know:

(3) Can the results in the present paper also be extended to d.g. near rings?

Once again, we propose the following:

(4) Does every member in a primary class of derivations have to be an inner derivation?

\section{REFERENCES}

1. I. N. Herstein, The structure of a certain class of rings, Amer. J. Math., 75 (1953), 864-871.

2. - A condition for the commutativity of rings, Canad. J. Math., 9 (1957), $583-586$.

3. - Noncommutative rings, John Wiley, N. Y., 1968.

4. N. Jacobson, Structure theory for algebras of bounded degree, Ann. of Math., 46 (1945), 695-707.

5. — Structure of rings, Amer. Math. Soc. Colloquium Publ. 37, rev. ed., Providence, 1964.

6. S. Ligh, The structure of certain classes of rings and near rings, J. London Math. Soc., (2), 12 (1975), 27-31.

7. Wallace S. Martindale, III, The commutativity of a special class of rings, Canad.

J. Math., 12 (1960), 263-268.

8. M. S. Putcha, R. S. Wilson and A. Yaqub, Structure of rings satisfying certain 
identities on commutators, Proc. Amer. Math., 32 (1972), 57-62.

9. Yuzo Utumi, On $\xi$-rings, Proc. Japan Acad., 33 (1957), 63-66.

Received February 21, 1978 and in revised form June 16, 1978.

North Carolina State University at Raleigh

RALEIGH, NC 27607 



\section{PACIFIC JOURNAL OF MATHEMATICS}

\section{EDITORS}

RICHARD ARENS (Managing Editor)

University of California

Los Angeles, California 90024

C. W. CURTIS

University of Oregon

Eugene, OR 97403

C. C. MOORE

University of California

Berkeley, CA 94720
J. DugundjI

Department of Mathematics University of Southern Californı Los Angeles, California 90007

R. Finn and J. Milgram Stanford University Stanford, California 94305

ASSOCIATE EDITORS

E. F. BECKENBACH

B. H. NeUmanN

F. WOLF

K. YoSHIDA

\section{SUPPORTING INSTITUTIONS}

UNIVERSITY OF BRITISH COLUMBIA CALIFORNIA INSTITUTE OF TECHNOLOGY

UNIVERSITY OF CALIFORNIA

MONTANA STATE UNIVERSITY

UNIVERSITY OF NEVADA, RENO

NEW MEXICO STATE UNIVERSITY

OREGON STATE UNIVERSITY

UNIVERSITY OF OREGON
UNIVERSITY OF SOUTHERN CALIFORNIA

STANFORD UNIVERSITY

UNIVERSITY OF HAWAII

UNIVERSITY OF TOKYO

UNIVERSITY OF UTAH

WASHINGTON STATE UNIVERSITY

UNIVERSITY OF WASHINGTON 


\section{Pacific Journal of Mathematics}

\section{Vol. 80, No. $1 \quad$ September, 1979}

Jeroen Bruijning and Jun-iti Nagata, A characterization of covering dimension by

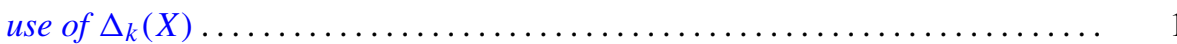

John J. Buoni and Albert Jonathan Klein, On the generalized Calkin algebra ...... 9

Thomas Ashland Chapman, Homotopy conditions which detect simple homotopy

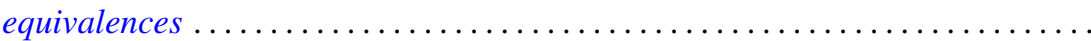

John Albert Chatfield, Solution for an integral equation with continuous interval

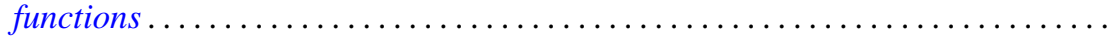

Ajit Kaur Chilana and Ajay Kumar, Spectral synthesis in Segal algebras on

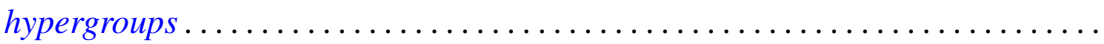

Lung O. Chung, Jiang Luh and Anthony N. Richoux, Derivations and

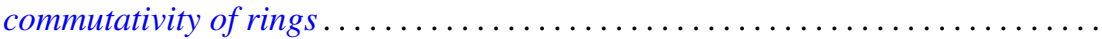

Michael George Cowling and Paul Rodway, Restrictions of certain function spaces to closed subgroups of locally compact groups .....................

David Dixon, The fundamental divisor of normal double points of surfaces........

Hans Georg Feichtinger, Colin C. Graham and Eric Howard Lakien,

Nonfactorization in commutative, weakly selfadjoint Banach algebras . . . . . . .

Michael Freedman, Cancelling 1-handles and some topological imbeddings ....... .

Frank E., III Gerth, The Iwasawa invariant $\mu$ for quadratic fields . . . . . . . . . . . . . .

Maurice Gilmore, Three-dimensional open books constructed from the identity

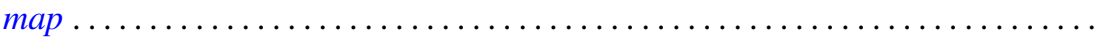

Stanley P. Gudder, A Radon-Nikodým theorem for $*$-algebras .

Peter Wamer Harley, III and George Frank McNulty, When is a point Borel? .

Charles Henry Heiberg, Fourier series with bounded convolution powers . .

Rebecca A. Herb, Characters of averaged discrete series on semisimple real Lie

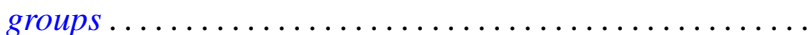

Hideo Imai, On singular indices of rotation free densities . .

Sushil Jajodia, On 2-dimensional CW-complexes with a single 2-cell . . .

Herbert Meyer Kamowitz, Compact operators of the form $u C_{\varphi}$

Matthew Liu and Billy E. Rhoades, Some properties of the Chebyshev method...

213

George Edgar Parker, Semigroups of continuous transformations and generating

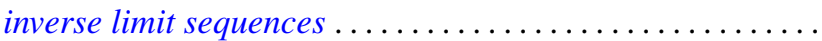

Samuel Murray Rankin, III, Oscillation results for a nonhomogeneous

equation ...

Martin Scharlemann, Transverse Whitehead triangulations ...

Gary Joseph Sherman, A lower bound for the number of conjugacy classes in a

finite nilpotent group

Richard Arthur Shoop, The Lebesgue constants for $\left(f, d_{n}\right)$-summability .

Stuart Jay Sidney, Functions which operate on the real part of a uniform

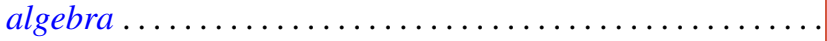

Tim Eden Traynor, The group-valued Lebesgue decomposition 